\title{
La declaración PRISMA 2020: una guía actualizada para reportar revisiones sistemáticas
}

\author{
The PRISMA 2020 statement: an updated guideline to report systematic reviews
}

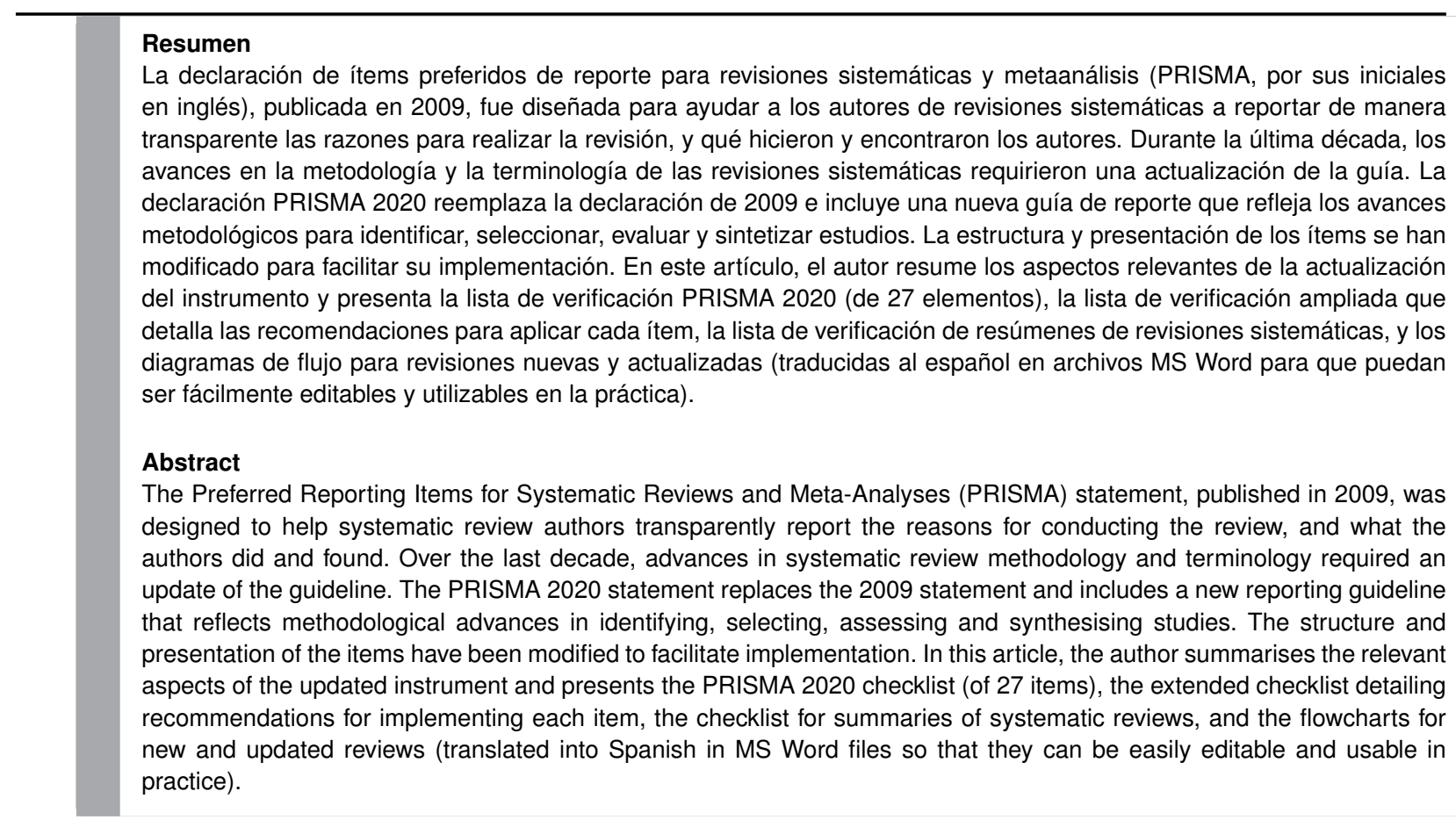

Palabras clave: Medicina Basada en la Evidencia, Revisiones Sistemáticas como Asunto. Keywords: Evidence-Based Medicine, Systematic Reviews as Topic.

\section{Traducido y resumido de:}

Page MJ, et al. Syst Rev. 2021;10(1):89. PMID: 337813481

\section{Introducción}

Las revisiones sistemáticas (RS) cumplen numerosos roles considerados esenciales. Pueden proporcionar una síntesis del estado del conocimiento en un campo, a partir del cual se pueden identificar las futuras prioridades de investigación, abordar preguntas que de otro modo no podrían ser respondidas por estudios individuales, identificar problemas en la investigación primaria que deberían rectificarse en estudios futuros y generar o evaluar teorías sobre cómo o por qué ocurren los fenómenos. Por lo tanto, las RS generan diversos tipos de conocimiento para los diferentes usuarios de las revisiones (como pacientes, prestadores de atención médica, investigadores y formuladores de políticas). Para garantizar que una RS sea valiosa para los usuarios, los autores deben preparar un reporte transparente, completo y preciso de por qué se realizó la revisión, qué hicieron ( $p$. ej., cómo identificaron y seleccionaron los estudios) y qué encontraron (como las características de los estudios incluidos y los resultados del meta-análisis).

La declaración PRISMA 2009 es una guía diseñada para mejorar el reporte deficiente de las $\mathrm{RS}^{2,3}$. Sus recomendaciones han sido ampliamente respaldadas y adoptadas por casi 200 revistas y organizaciones de RS, y su adopción y uso en varias disciplinas se asocia con reportes de RS más completos ${ }^{4}$.

Los avances metodológicos, los cambios de terminología de la evaluación de la çalidad"hacia la evaluación de la çerteza.en el cuerpo de evidencia ${ }^{5}$ y la facilidad actual para registrar y difundir protocolos de RS, exigía una actualización de la declaración PRISMA. Los detalles de su desarrollo fueron presentados recientemente ${ }^{6}$.

\section{Alcance de la declaración PRISMA 2020}

La declaración PRISMA 2020 fue diseñada principalmente para evaluar RS de estudios que evalúan los efectos de intervenciones de salud, con independencia del diseño de los estudios incluidos. Sin embargo, es aplicable a reportes de RS que evalúan otros tipos de intervenciones (como sociales o educativas), y muchos de sus ítems son aplicables a RS que tienen objetivos diferentes a los de la evaluación de intervenciones (como etiología, prevalencia o pronóstico). PRISMA 2020 está diseñada para ser utilizada tanto en RS que incluyen síntesis (como meta-análisis u otros métodos de síntesis estadística) como en aquellas que no la incluyen. PRISMA 2020 se puede utilizar para RS originales, RS actualizadas o RS actualizadas de manera permanente (llamadas revisiones vivas). Las definiciones de algunos de los términos utilizados en PRISMA 2020 y en el diagrama de flujo propuesto son importantes en particular, por lo que se detallan a continuación.

1. Reporte: documento (en papel o electrónico) que proporciona información sobre un estudio en particular. Puede ser un artículo de revista, una versión preliminar, un resumen de una conferencia, el registro de un estudio, el reporte de un estudio clínico (clinical study report), una disertación, un manuscrito no publicado, un reporte gubernamental u otro documento que proporcione información relevan- 


\section{te.}

2. Registro: título y/o resumen de un reporte indizado en una base de datos o sitio web (como Medline). Los registros que se refieren al mismo reporte (como el mismo artículo de revista) son considerados duplicados, sin embargo, los que se refieren a reportes similares (como los enviados a dos conferencias diferentes) deben considerarse únicos.

3. Estudio: una investigación (como un ensayo clínico), que incluye un grupo definido de participantes, una o más intervenciones y sus resultados. Un estudio puede tener varios reportes. Por ejemplo, los reportes podrían incluir el protocolo, el plan de análisis estadístico, las características iniciales, los resultados del objetivo primario, los resultados de los daños, los resultados de los objetivos secundarios y los resultados de los análisis adicionales de mediadores y moderadores.

PRISMA 2020 no tiene la intención de guiar la realización de RS, para ello existen otros recursos disponibles ${ }^{7,8}$. Sin embargo, puede resultar útil para asegurar que se capture toda la información recomendada durante la realización de una RS. PRISMA 2020 tampoco debe utilizarse para evaluar la realización o la calidad metodológica de la RS, para lo cual también existen otras herramientas ${ }^{9,10}$. Se han desarrollado múltiples extensiones de la declaración PRISMA 2009 (disponibles en la red EQUATOR). Para este tipo de revisiones, recomendamos a los autores que reporten su revisión de acuerdo con las recomendaciones de PRISMA 2020 junto con la guía específica para la extensión.

\section{Cómo usar PRISMA 2020}

La declaración PRISMA 2020 (incluidas las listas de verificación, la explicación, la elaboración y el diagrama de flujo) reemplaza a la declaración PRISMA 2009, que ya no debe utilizarse. La Tabla 1 resume los principales cambios realizados a la declaración PRISMA 2009.

Es recomendable que los autores consulten PRISMA 2020 al principio del proceso de realización de una RS, ya que la consideración prospectiva de sus ítems puede ayudar a garantizar que se aborden todos ellos. El sitio web de la declaración PRISMA (http ://www.prisma-statement.org/) contiene plantillas de las listas de verificación en inglés para descargar y completar. Existe además una aplicación web con una interfaz de fácil uso, que permite a los usuarios completar la lista de verificación ingresando a https://pri sma.shinyapps.io/checklist/ y exportarla a MS Word o PDF. Esta plantilla permite localizar la ubicación de la información, pero el principio rector es garantizar que la información sea presentada.

El diagrama de flujo describe el flujo de información de las diferentes fases de una RS. Traza el número de registros identificados, el número de estudios incluidos y excluidos, y las razones de las exclusiones. La Figura 1 muestra el diagrama de flujo de PRISMA 2020 para RS según el tipo de revisión (nueva o actualizada) y las fuentes utilizadas para identificar los estudios. En el sitio web de declaraciones de PRISMA (http://www.prisma-state ment.org/PRISMAStatement/FlowDiagram) se encuentran disponibles diferentes plantillas descargables en inglés. En el material suplementario se encuentran disponibles las distintas versiones en español.
Tabla 1. Principales cambios del PRISMA 2020 con respecto a la declaración PRISMA de 2009

- Inclusión de una lista de verificación de resúmenes ${ }^{11}$ (ítem \# 2).

- El ítem "Protocolo y registro" agrega un sub-ítem que recomienda que los autores describan las enmiendas de la información proporcionada en el registro o en el protocolo (ítem \# 24a -24c).

- La modificación del ítem de "Búsqueda" para que los autores presenten las estrategias de búsqueda completas para todas las bases de datos, los registros y los sitios web, en lugar de la estrategia utilizada en una sola base de datos (ítem \# 7).

- Modificación del ítem "Selección de estudios" de la sección de Métodos para enfatizar el reporte de cuántos revisores examinaron cada registro y/o reporte recuperado, si lo hicieron de forma independiente y, si corresponde, los detalles de las herramientas de automatización utilizadas en el proceso (ítem \# 8).

- La adición de un sub-ítem denominado "Ítems de datos"que recomienda informar cómo se definieron los resultados, qué resultados se buscaron y qué métodos se utilizaron para seleccionar un subconjunto de resultados de los estudios incluidos (ítem \# 10a).

- La división del ítem "Síntesis de resultados" de la sección de Métodos en seis sub-ítems donde se recomienda describir: los procesos utilizados para decidir qué estudios eran elegibles para cada síntesis, los métodos necesarios para preparar los datos para la síntesis, los métodos utilizados para tabular o mostrar visualmente los resultados de estudios individuales o síntesis, los métodos utilizados para sintetizar los resultados, los métodos utilizado para explorar las posibles causas de heterogeneidad entre los resultados de los estudios (como análisis de subgrupos, metarregresión); y el análisis de sensibilidad utilizado para evaluar la robustez de los resultados sintetizados (ítem \# 13a-13f).

- La adición de un sub-ítem al ítem "Selección de estudios" de la sección Resultados, recomendando a los autores citar estudios que aparentan cumplir con los criterios de inclusión, pero que fueron excluidos, explicando por qué fueron excluidos (ítem \# 16b).

- División del ítem "Síntesis de resultados" de la sección Resultados en cuatro sub-ítems que recomiendan a los autores: resumir brevemente las características y el riesgo de sesgo entre los estudios que contribuyen a la síntesis, presentar los resultados de todas las síntesis estadísticas realizadas, presentar los resultados de la investigación de posibles causas de heterogeneidad entre resultados de los estudios y presentar los resultados de los análisis de sensibilidad (ítem \# 20a-20d).

- Adición de nuevos ítems que recomiendan a los autores reportar los métodos y resultados de una evaluación de certeza (o confianza) en el cuerpo de evidencia para un resultado (ítems \# 15 y \# 22).

- Adición de un nuevo ítem que recomienda a los autores declarar cualquier conflicto de interés (ítem \# 26).

- La adición de un nuevo ítem para indicar si los datos, el código analítico y otros materiales utilizados en la revisión están disponibles públicamente y, de ser así, dónde se los puede encontrar (ítem \# 27).

El diagrama de flujo describe el flujo de información de las diferentes fases de una RS. Traza el número de registros identificados, el número de estudios incluidos y excluidos, y las razones de las exclusiones. La Figura 1 muestra el diagrama de flujo de PRISMA 2020 para RS según el tipo de revisión (nueva o actualizada) y las fuentes utilizadas para identificar los estudios. En el sitio web de declaraciones de PRISMA (http://www.prisma-state ment.org/PRISMAStatement/FlowDiagram) se encuentran disponibles diferentes plantillas descargables en inglés. En el material suplementario se encuentran disponibles las distintas versiones en español.

En otra publicación se brinda una extensa explicación del desarrollo y forma de aplicación de cada ítem ${ }^{12}$. En el material suplementario se encuentra disponible la lista de verificación ampliada y su versión abreviada de los ítems presentados en dicho documento. 
Las revistas y los editores pueden imponer límites de palabras, secciones y/o cantidad de tablas y figuras permitidas en el reporte principal. En tales casos, si la información relevante para algunos ítems ya aparece en un protocolo de revisión de acceso públi$\mathrm{co}$, puede ser suficiente con citar el protocolo. Alternativamente se recomienda colocar descripciones detalladas de los métodos utilizados o resultados adicionales (por ejemplo, para los resultados menos importantes) en archivos suplementarios que idealmente deben encontrarse disponibles en un repositorio de acceso abierto, gratuito y permanente (como Open Science Framework, Dryad, Figshare). Se debe incluir una referencia o enlace a la información adicional en el reporte principal.

Figura 1. Plantilla PRISMA 2020 de diagrama de flujo para revisiones sistemáticas. Notas: Los cuadros en gris solo deben completarse si correspondiera; de lo contrario, deben eliminarse del diagrama de flujo. Tenga en cuenta que un "reporte" puede ser un artículo de revista, un artículo preimpreso, un resumen de una conferencia, un estudio inscripto en un registro, un reporte clínico de estudio, una disertación, un manuscrito no publicado, un informe de gobierno o cualquier otro documento que proporcione información relevante. Considere si es posible informar el número de registros identificados de cada base de datos o registro buscado (en lugar del número total en todas las bases de datos / registros).

Si se usaron herramientas de automatización, indique cuántos registros fueron excluidos por un ser humano y cuántos fueron excluidos por las herramientas de automatización.

\section{Estudios previos} anterior de la revisión ( $\mathrm{n}=$ ) Informes de estudios incluidos en la versión anterior de la revisión ( $n=)$

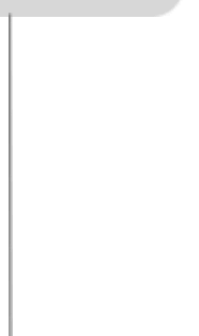

Identificación de nuevos estudios vía bases de datos y registros

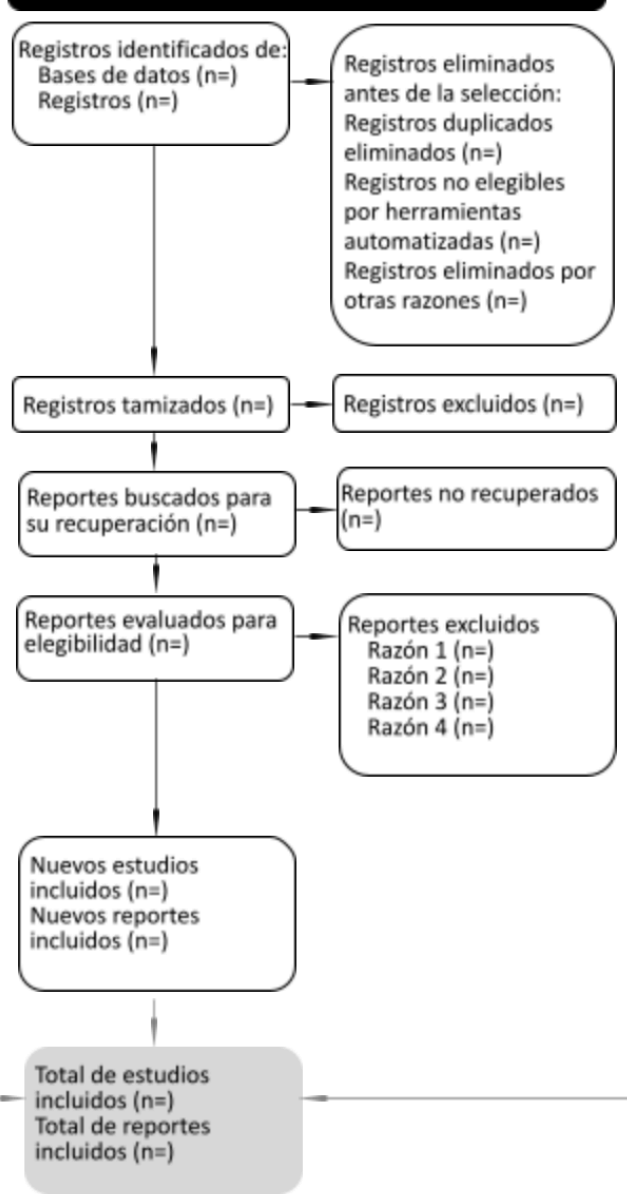

Identificación de nuevos estudios vía otros métodos

Registros identificados de

Sitios web $(n=)$

Organizaciones $(n=)$

Búsqueda de citas $(n=)$

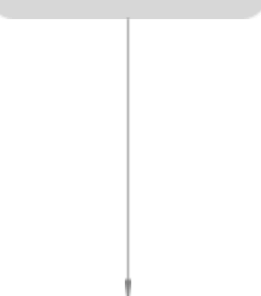

Reportes buscados para su recuperación ( $n=)$

Reportes no recuperados $(n=)$

\section{Reportes evaluados para} elegibilidad $(n=)$

\section{Relevancia}

La declaración PRISMA 2020, desarrollada por un amplio y representativo grupo de expertos, tiene el potencial de beneficiar a múltiples actores entre los que se encuentran autores, editores y revisores de revistas y usuarios de las revisiones (desarrolladores de guías, formuladores de políticas, prestadores de atención médica o pacientes). Los reportes completos permiten a los lec- tores evaluar la idoneidad de los métodos y, por lo tanto, la confiabilidad de los hallazgos. Por ejemplo, la introducción de la certeza en el cuerpo de evidencia para cada resultado y las implicancias de los hallazgos ayudaría a los encargados de formular políticas de salud, a los administradores y a otros tomadores de decisiones, a formular recomendaciones apropiadas para la práctica clínica o la política sanitaria. A su vez, facilita tanto la replicación y 
la actualización de las RS, como su inclusión en revisiones panorámicas de $\mathrm{RS}$ (<italic id=.e-3c43b30ed2b2">overviews</italic>) y guías de práctica clínica. Se han propuesto varias estrategias para aumentar el uso de las guías de reporte incluyendo a los educadores, que presentan pautas para la presentación de informes en los planes de estudio de posgrado, los editores de revistas, que respaldan el uso de directrices de presentación de informes, los revisores de pares, que evalúan el cumplimiento de las recomendaciones, y los autores, que los utilizan durante la etapa de redacción o, de forma ideal, desde el diseño del estudio. Es esperable que la adopción de esta guía conduzca a la disponibilidad de reportes más transparentes, completos y precisos de las $\mathrm{RS}$, que faciliten así la toma de decisiones basadas en la evidencia.

\section{Material suplementario}

Como material suplementario se encuentran disponibles en este artículo las siguientes plantillas en español:

1. Diagrama de flujo de PRISMA 2020 para nuevas RS que incluyen únicamente búsquedas en bases de datos y registros.

2. Diagrama de flujo de PRISMA 2020 para nuevas RS que incluyen búsquedas en bases de datos, registros y otras fuentes.

3. Diagrama de flujo de PRISMA 2020 para RS actualizadas que incluyen únicamente búsquedas en bases de datos y registros.

4. Diagrama de flujo de PRISMA 2020 para RS actualizadas que incluyen búsquedas en bases de datos, registros y otras fuentes.

5. Lista de verificación PRISMA 2020.

6. Lista de verificación ampliada PRISMA 2020

7. Lista de verificación de PRISMA para resúmenes de revisiones sistemáticas.

Fuente de financiamiento/Conflicto de interés de los autores: Esta investigación no contó con financiamiento directo. Sus autores declararon sus fuentes de financiamiento propias antes y durante el desarrollo de este trabajo, las contribuciones realizadas al estudio y sus potenciales conflictos de interés como parte del equipo editorial de varias revistas biomédicas y/o entidades dedicadas a la síntesis de evidencia.

Recibido el 09/04/2021 Aceptado el 19/07/2021 y Publicado el 04/08/2021.

Agustín Ciapponi [ Servicio de Medicina Familiar y Comunitaria, Hospital Italiano de Buenos Aires; Centro Cochrane Argentina, Instituto de Efectividad Clínica y Sanitaria. aciapponi@iecs.org.ar ]

Ciapponi A. La declaración PRISMA 2020: una guía actualizada para reportar revisiones sistemáticas. Evid Actual Pract Ambul. $2021 ; 24(3)$ :e002139. Available from: https://dx.doi.org/10.51987/EVIDENCIA.V24I4.6960. Comentado de: Page MJ, et al. The PRISMA 2020 statement: an updated guideline for reporting systematic reviews. Syst Rev. 2021 Mar 29;10(1):89. PMID: 33781348

\section{Referencias}

1. Page MJ, Mckenzie JE, Bossuyt PM, et al. The PRISMA 2020 statement: an updated guideline for reporting systematic reviews. Syst Rev. 2021;10(1):89-89. Available from: 10.1186/s13643-021-01626-4.

2. Moher D, Liberati A, Tetzlaff J, , et al. Preferred Reporting Items for Systematic Reviews and Meta-Analyses: The PRISMA Statement. PLoS Med. 2009;6(7):e1000097-e1000097. Available from: 10.1371/journal.pmed.1000097;https://dx.doi.org/10.1371/journal.pmed.1000097.

3. Ciapponi A. Novedades en el reporte (PRISMA) y en la evaluación de la calidad (AMSTAR) de las revisiones sistemáticas y los meta-análisis. Evid Actual Pract Ambul. 2009;12(4):141-145. Available from: 10.51987/EVIDENCIA.V12I4.5820;http://www.evidencia.org/index.php/Evidencia/article/ view/5820/3383.

4. Page MJ, Shamseer L, Altman DG, et al. Epidemiology and Reporting Characteristics of Systematic Reviews of Biomedical Research: A CrossSectional Study. PLOS Med. 2016;13(5):e1002028-e1002028. Available from: 10.1371/journal.pmed.1002028;https://dx.doi.org/10.1371/journal. pmed.1002028.

5. Hultcrantz M, Rind D, Akl EA, et al. The GRADE Working Group clarifies the construct of certainty of evidence. J Clin Epidemiol. 2017;87:4-13. Available from: 10.1016/j.jclinepi.2017.05.006;https://dx.doi.org/10.1016/j.jclinepi.2017.05.006

6. Page MJ, McKenzie JE, Bossuyt PM, et al. Updating guidance for reporting systematic reviews: development of the PRISMA 2020 statement. J Clin Epidemiol. 2021;134:103-112. Available from: 10.1016/j.jclinepi.2021.02.003;https://dx.doi.org/10.1016/j.jclinepi.2021.02.003.

7. Dekkers OM, Vandenbroucke JP, Cevallos M, et al. COSMOS-E: Guidance on conducting systematic reviews and meta-analyses of observational studies of etiology. PLOS Med. 2019;16(2):e1002742-e1002742. Available from: 10.1371/journal.pmed.1002742;https://dx.doi.org/10.1371/journal. pmed.1002742.

8. Higgins J, Thomas J, Chandler J, et al. Cochrane Handbook for Systematic Reviews of Interventions version 6.0 (updated August 2019 ). Cochrane; 2019. Available from: https://training.cochrane.org/handbook.

9. Whiting P, Savović J, Higgins JPT, et al. ROBIS: A new tool to assess risk of bias in systematic reviews was developed. J Clin Epidemiol. 2016;69:225234. Available from: 10.1016/j.jclinepi.2015.06.005;https://dx.doi.org/10.1016/j.jclinepi.2015.06.005.

10. Shea BJ, Reeves BC, Wells G, et al. AMSTAR 2: a critical appraisal tool for systematic reviews that include randomised or non-randomised studies of healthcare interventions, or both. BMJ;358:j4008-j4008. Available from: 10.1136/bmj.j4008;http://dx.doi.org/10.1136/bmj.j4008.

11. Beller EM, Glasziou PP, Altman DG, et al. PRISMA for Abstracts: reporting systematic reviews in journal and conference abstracts. PLoS Med. 2009;10(4):1001419-1001419. Available from: 10.1371/journal.pmed.1001419.

12. Page MJ, Moher D, Bossuyt PM, et al. PRISMA 2020 explanation and elaboration: updated guidance and exemplars for reporting systematic reviews. BMJ. 2021;372:n160-n160. Available from: 10.1136/bmj.n160. 